LM

$26,4 / 5$

206

\section{Towards personalization in digital libraries through ontologies}

\author{
Núria Ferran
}

Digital Library, Universitat Oberta de Catalunya (UOC), Barcelona, Spain, and Enric Mor and Julià Minguillón

Computer Science and Multimedia Studies, Universitat Oberta de Catalunya (UOC), Barcelona, Spain

\begin{abstract}
Purpose - To describe a browsing and searching personalization system for digital libraries based on the use of ontologies for describing the relationships between all the elements which take part in a digital library scenario of use.

Design/methodology/approach - Identification of all the desired functionalities and requirements that are necessary to fully integrate the use of a digital library in an e-learning environment, and the basic elements that are used to build the ontology that describes such scenario.

Findings - The elements that determine the functionalities of the desired personalization system: first, the user's profile, including navigational history and user preferences; and second, the information collected from the navigational behavior of the digital library users.

Research limitations/implications - The ontology is not complete. In fact, the ontology in itself will evolve with the new apparition of desired functionalities and requirements of the personalization system.

Practical implications - Such a personalization system will be very helpful to the users of a digital library to improve their experience of use.

Originality/value - The use of ontologies promotes the integration of new services into existing ones, and the interoperability with other systems through the appropriate semantic web services. New system functionalities and requirements can be added by including the appropriate description into the ontology framework that defines the digital library scenario of use.
\end{abstract}

Keywords Digital libraries, Information searches, Personal needs, Customization, Distance learning

Paper type Research paper

\section{Introduction}

Distance education is becoming one of the most attractive methods for incorporating all kinds of people into higher and university degree education levels, moving towards a "blended" technology approach deploying multiple technologies. The introduction of new technologies of information and communications with the intensive use of e-learning environments, as a virtual campus, for example, allows students to break through the barriers of space and time, and to design their own lifelong curricula, adapting it to their particular necessities and preferences, according to their possibilities as students, changing the usual way of both teaching and learning (Jonassen et al., 1995), setting up the foundations of e-learning environments (Rosenberg, 2001).

The students of an e-learning environment have access to a predetermined \begin{abstract}
repository of learning resources, which are part of the learning process designed by the
\end{abstract}
Library Management Vol. 26 No. 4/5, 2005 pp. 206-217

(C) Emerald Group Publishing Limited 0143-5124

DOI 10.1108/01435120510596062 
team of instructional designers and teachers for each course. But usually, these students might need more additional learning resources and documents to successfully follow the recommended learning itineraries, so there is the necessity of providing mechanisms for accessing such resources in a structured manner (Sicilia and García, 2005). On the other hand, researchers and teachers in an e-learning environment are also usual "customers" of the services offered by an academic library, although under a completely different approach. These kinds of users have different experiences of use of the digital library than students, as their goals are clearly different, in both content and context aspects. All this richness needs to be captured somehow in order to better understand the way users of a digital library perform their navigation. Digital libraries must evolve in order to be proactive, more responsive to possible changes and to include new services such as personalization in order to increase user satisfaction and fidelity.

Therefore, in an ideal scenario, the digital library should adapt to the specific characteristics of each user profile, but also to the particular necessities and preferences of each user, combining both user and profile level personalization capabilities. Personalization is one of the key factors which are directly related to user satisfaction (Riecken, 2000) and, therefore, linked to the failure or success of the performed activity, although it must be carefully introduced (Nielsen, 1998). Personalization has been shown useful in several areas such as e-commerce (Kasanoff, 2001), business-to-business companies (Colkin, 2001), and obviously reproduced in other environments such e-learning (Mor and Minguillón, 2004), for example. Regarding the library management field, there exist remarkable approaches such as the recommendation system for electronic journals of MyLibrary from Los Alamos National Laboratory 2.4 (http://lib-www.lanl.gov/) or MyOpenLibrary from the Open University (http://library.open.ac.uk/index.html); such personalization librarian initiatives are showing successful results as appears at the research being done about the user satisfaction (Sinha and Swearingen, 2001).

In order to build such personalization system, several multidisciplinary aspects must be addressed: first, there are cognitive and behavioral aspects (Ford and Ford, 1993) that determine the way users perform searches and examine the obtained results. The "I'm Feeling Lucky" button in the Google search engine home page is a good example of such fact. Second, personalization issues must be addressed from a user-centered point of view, under the approach of human computer interaction, as it is well known (Schonberg et al., 2000) that most personalization systems fail, not because of the personalization system in itself, but in the interaction with the user and the way recommendations are presented. Third, there are technological and knowledge engineering aspects related to the way all this information is structured for both updating and querying purposes. In this paper we describe the set of desired functionalities and requirements of an ideal scenario for a digital library which includes personalization capabilities by means of ontologies. The use of ontologies for describing the possible scenarios of use in a digital library brings the possibility of predicting user requirements in advance and to offer personalized services ahead of expressed need. Ontologies are built using other sub-ontologies which describe the basic elements of the personalization system: users, digital resources, actions, navigational profiles, etc. This diversity of elements suggests that there is a need for further focus on the interoperability of objects which in turn requires well developed 
LM

$26,4 / 5$

208 ontologies to describe the properties of both objects and individuals and the relationships between them (Brophy, 2004).

This paper is structured as follows: the second section describes the basic functionalities of a digital library and the specific case of the UOC digital library, and the fundamental concepts of ontologies used in this paper. The third section defines the requirements and functionalities of a personalized digital library integrated in a virtual e-learning environment. All the elements defined as part of the ontology which gives support to the personalization system are described in the fourth section. Finally, conclusions and future research directions are outlined in the fifth section.

\section{Digital libraries and the case of the UOC}

As stated previously, one of the most important resources for supporting users in a distance e-learning environment is the possibility of accessing to a digital library, which allows the users to collect and organize the necessary information for achieving their particular goals. Furthermore, the search of information can be a learning but also an assessment activity by itself, so it is important to ensure and facilitate a proper use of the library.

There are several terms being used interchangeably when we approach the concept of a library with digitized data and accessible remotely. Among these we can find hybrid library, digital library and virtual library. An informal definition of a digital library is "a managed collection of information, with associated services, where the information is stored in digital formats and accessible over a network" (Arms, 2002). The hybrid library provides electronic information sources too but also paper-based information. The hybrid should be considered as a model by itself not as a transitional phase from a conventional library to a digital one (Brophy, 2001). And finally the virtual library has been defined as the concept of a "remote access to the contents and services of libraries and other information resources, combining an on-site collection of current and heavily used materials in both print and electronic form, with an electronic network which provides access to, and delivery from, external worldwide library and commercial information and knowledge sources" (Gapen, 1993).

Nowadays, we are finding new types of libraries coming up from long-term personal digital libraries, as well as digital libraries that serve specific organizations, educational needs, and cultural heritage and that vary in their reliability, authority and quality. Besides, the collections are becoming more heterogeneous in terms of their creators, content, media, and communities served. In addition, the user communities are becoming heterogeneous in terms of their interests, backgrounds, and skill levels, ranging from novices to experts in a specific subject area (Callan and Smeaton, 2003). This growing diversity has changed the initial focus of providing access to digital content and transforming the traditional services into digital ones to a new handicap where the next generation of libraries should be more proactive offering personalized information to their users taking in consideration each person individually (his or her goals, interests, level of education, etc.).

While data and information are captured and represented in various digital formats, and rapidly proliferating, the techniques for accessing data and information are rudimentary and imprecise, mostly based on simple keyword indexes, relational queries, and/or low-level image or audio features (i.e., research results of the 1970s and 1980s). In the current context of explosive availability of data, there is a need for a knowledge discovery approach, based on both top-down knowledge creation (e.g., 
ontologies, subject headings, user modeling) and bottom-up automated knowledge extraction (e.g., data mining, text mining, web mining), promises to help transfer digital library from an institution of data and information to an institution of knowledge (Chen, 2003).

\section{The UOC virtual library}

The UOC Library was born in 1995 as a virtual academic library to support a virtual e-learning university model and, since then, provides online services and information resources both print and digital, owned by the library or by other libraries. Users can access the library from any computer and do not need to move in order to get any information resource. All authorized users are able to use remotely the exclusive content of the library as subscribed databases like the Electronic Management Research Library Database (Emerald) or the Web of Knowledge from ISI and benefit from the services of the library such as the Selective Diffusion of Information, Bibliographic Searches or Loans, all of them performed through the web.

The UOC virtual library can be accessed in different ways. One of them is from the virtual campus where the user finds the whole content and services of the library. But the main focused (i.e., guided) entrance to the library can be found in the campus's virtual classrooms where teachers and librarians bring a selection of the most interesting resources for every subject, for instance the learning material, recommended bibliography where each book is linked with the loan form, electronic articles, self-assessment exercises, a selection of internet resources, databases and electronic journals, e-books, exams from previous years, etc. This has been the first step for tailoring information for a very well defined community.

The main objective of the library is to provide the students, but also lecturers, researchers and management staff, access to the information relevant for the fulfillment of their basic functions: learning, teaching, research and management. Several user profiles can be identified: undergraduate student, $\mathrm{PhD}$ student, teacher, learning process manager, among others. Each profile can be partially identified by the tasks related to the digital library that it performs. For example, students usually browse the digital library looking for documents related to activities such as exercises, exams and recommended articles and in very specific periods of time, when the delivery date of the exercise is due. On the other hand, teachers can navigate among the content of the library in order to mentor a student in doing his or her homework or providing content to the digital library associated to the virtual classroom. A final example could be the researchers who usually perform more focused searches during a larger period of time. Each of these users may have common goals in certain times but their knowledge, tasks, social activities and preferences are totally different.

\section{Ontologies and the Semantic Web}

Therefore, it is necessary to build a complete and complex structure for describing all the richness of the possible scenarios of use of the digital library and the relationships which can be established among all the participants. This can be achieved by means of ontologies and the use of the Semantic Web services in their appropriate forms (Sheth et al., 2005).

An ontology is, taking the meaning adopted by the semantic web community, a formal description of a possible scenario or context; that is, what "exists" is what can be represented by an ontology. Formally, an ontology is the statement of a logical 
LM

$26,4 / 5$

210 theory, but by "formal description" we also mean that it can be automatically queried and updated, as the main users of ontologies are (or should be) computers, not humans, in order to explicitly represent the objects, concepts and other entities that are assumed to exist in some context, altogether with the relationships that hold among them, although ontologies must be also human-readable. "Ontologies and taxonomies are, in functional terms, often used as synonyms. Computer scientists call hierarchies of structured vocabularies ontologies and librarians deploy the term taxonomy" (Adams, 2002). Nevertheless, ontologies also include a set of semantic rules which are used to infer knowledge from a structured hierarchy of information, giving to the complete structure a semantic meaning, not only syntactic (Gruber, 1995).

\section{Requiriments for an integrated, personalized digital library}

The web has become a very common tool for information browsing and searching, and the success of search engines such as Google or A9, for example, has facilitated the diffusion and access to repositories of digital documents. Despite that, one of the main problems of such search engines is that the generated results are not always of interest for the users performing the search, as these engines use a generalist approach based on several criteria which might not match the criteria of a specific user. On the other hand, several e-commerce web-based services, such as Amazon or e-Bay, for example, also provide browsing and searching services, but focused on categories. Both approaches can be combined to facilitate the way users browse the contents in search of information.

Several techniques are used for guidance and for providing recommendations to users; among others, collaborative filtering (Herlocker et al., 2004) is one of the most successful ones. Briefly, collaborative filtering is selecting content based on the preferences of people with similar interests, basically by pooling and ranking informed opinions (or experiences of use) on any particular topic. That is to say, an automatic system collects information about user actions (explicit, such as voting or answering a question; or implicit, such as noticing which offered links are visited and which are not, and how much time) and determines the relative importance of each content by weighting all the collected information among the large amount of users.

Both navigational techniques are also valid in a digital library scenario of use: simple searches starting from a single search term or advanced searches using multiple criteria, but also a recommendation system based on guided navigation through an ordered set of categories. The basic idea of this paper is that the efforts for finding a useful piece of information in a digital library carried out by an individual can be stored in a structured way and then shared for future users with similar necessities. Furthermore, if such information searching and browsing combines several web-based resources with different approaches (access mechanisms, query languages and interfaces, and so on), it is important to describe a common strategy (Sadeh and Walker, 2003) for minimizing the necessary efforts to fight against duplication (thus inconsistency) and source diversity.

\section{Identification of personalization system functionalities}

Two elements determine the functionalities of the desired personalization system: first, the user's profile, including navigational history and user preferences; and second, the information collected from the navigational behavior of the digital library users. User profile should include all the information relevant to user: personal information, which 
can be publicly made available by each user in order to facilitate the discovery of similar interests; and navigational history and behavior records, which will be used altogether with the personal information by the personalization system to build the set of recommendations that will help each user in browsing and searching the digital library. This information should help the user to improve his or her searches, by obtaining additional information when searching or browsing. It is remarkable to say that this information has been validated by the ontology, and that is not biased by any non-academic purpose of use (such as commercial-supported recommendations in Google or Amazon, for example). Table I shows the basic user profile attributes which are used to build the user model. For each attribute, it is shown whether it can be obtained or it must me given by the user, the relative importance for navigation and the recommendation system, and the user profiles which is more related to.

For instance, the socio-demographical attribute may include information about the languages spoken or understood by the user, information that can be used as an additional filtering when browsing or searching for documents. This attribute has a medium importance with respect to personalization of navigational actions, and a low importance for the recommendation system. On the other hand, other attributes which express user preferences and interests have a high importance for both navigational and recommendation system actions. Other information about profiles such as information about factual/behavioral issues (Adomavicius and Tuzhilin, 1999) can be also included at this level.

Two different behavior types can be identified, depending on the users' navigation, exploratory navigation and goal-oriented navigation. The exploratory navigation can be mainly oriented to obtain a general vision of the available resources in the library. Depending on user profile, the exploratory navigation would have different implicit intentions. In the case of goal-oriented navigation, it is usually considered that the user is looking for a resource. These searches can be classified in different use cases. For example, in the situation of searching for an author, if the user is a student, the

\begin{tabular}{|c|c|c|c|c|c|}
\hline Attributes & $\begin{array}{l}\text { Is it } \\
\text { explicit? }\end{array}$ & $\begin{array}{l}\text { Navigational } \\
\text { support } \\
\text { value level }\end{array}$ & $\begin{array}{l}\text { Library } \\
\text { recommendation } \\
\text { level }\end{array}$ & $\begin{array}{l}\text { Profiles } \\
\text { concerned }\end{array}$ & \\
\hline $\begin{array}{l}\text { Academic Register, Actual } \\
\text { enrollment }\end{array}$ & No & High & High & Student & \\
\hline $\begin{array}{l}\text { Academic Register, Previous } \\
\text { enrollment }\end{array}$ & No & Low & High & Student & \\
\hline Navigational history & No & High & Medium & All & \\
\hline $\begin{array}{l}\text { Navigational behavior and } \\
\text { strategies }\end{array}$ & No & High & Medium & All & \\
\hline $\begin{array}{l}\text { Socio-demographical profile, } \\
\text { General background }\end{array}$ & No & Medium & Low & All & \\
\hline $\begin{array}{l}\text { Socio-demographical profile, } \\
\text { Academic background }\end{array}$ & No & Low & Medium & Student & \\
\hline Knowledge area & Yes & High & High & $\begin{array}{l}\text { Teacher, } \\
\text { Consultant, } \\
\text { Researcher }\end{array}$ & Table I \\
\hline Interests & Yes & High & High & All & Basic user profile \\
\hline Preferences & Yes & High & High & All & attributes \\
\hline
\end{tabular}


LM

$26,4 / 5$

212

recommendations associated to search results should be oriented to the area of the course subjects, taking into account the navigation of other students and also the recommendations of the teachers. If the user is a researcher, recommendations should be oriented by different criteria depending on the searches that have been carried out by other investigators, or to the magazines, books and conferences where the searched author had published, understanding that the same magazine, conference or book might contain other interesting resources. Recommendations are generated using the knowledge extracted from the searching and browsing profiles of users with similar interests, knowledge integrated in the ontology such as course bibliography, or by following citations of similar documents, for example. Regarding the sources of information, using the library for accessing selected free Internet resources will be of particular interest, because the recommendation system ensures the users receive the opinions of a large set of experts (that is, the collaborative filtering system), therefore giving authenticity to such electronic information sources. On the other hand, regarding the library exclusive content, the user will get into the external databases (commercial) or internal databases (such as digital repositories or catalogues) in a transparent manner. Table II shows all the current information sources present in the digital library, and the basic actions that can be taken by the library users.

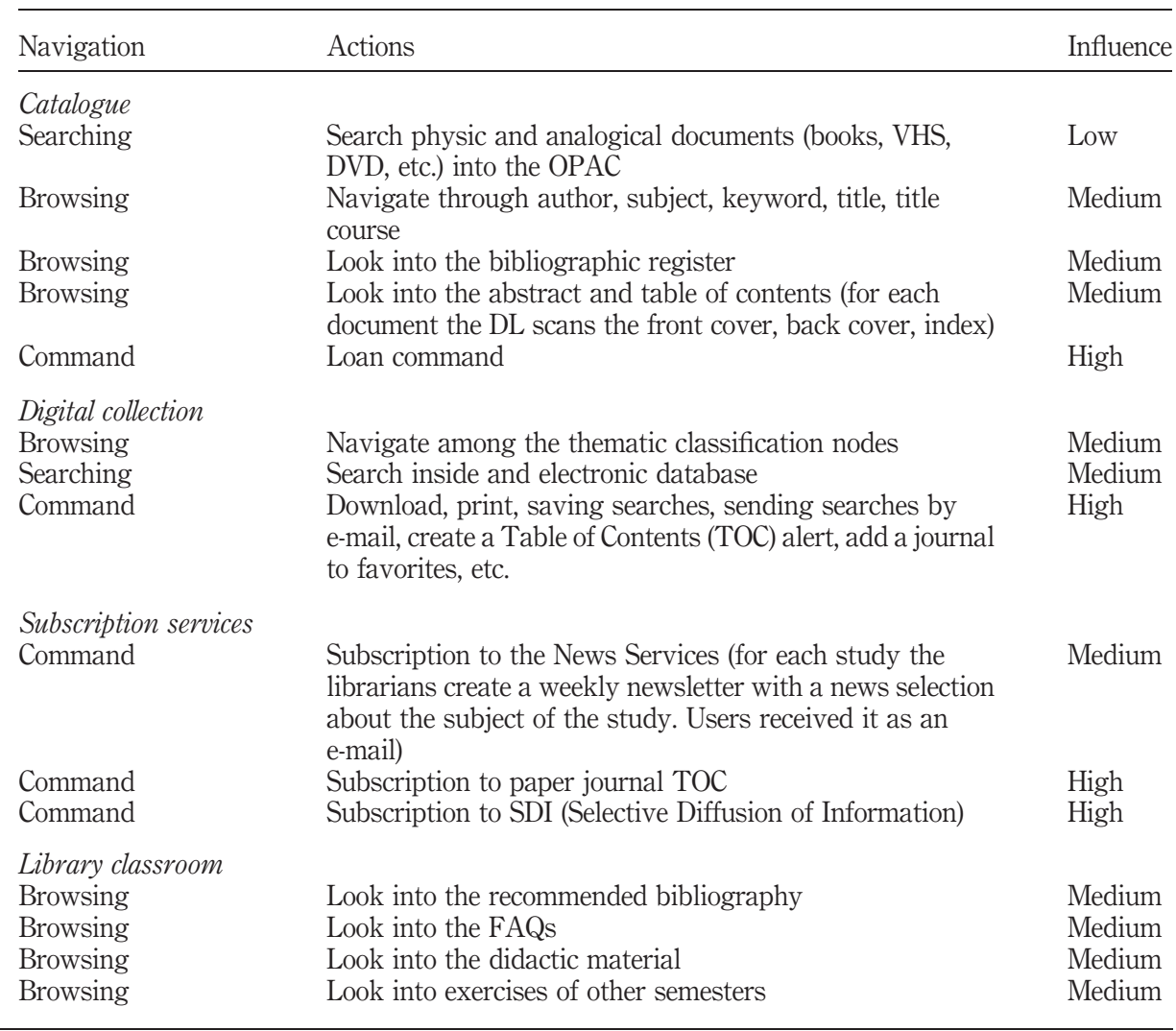

Table II.

Information sources and basic user actions in the digital library
Search physic and analogical documents (books, VHS,

Navigate through author, subject, keyword, title, title course

document the DL scans the front cover, back cover, index)

Loan command

Navigate among the thematic classification nodes

Search inside and electronic database

Download, print, saving searches, sending searches by

e-mail, create a Table of Contents (TOC) alert, add a journal

to favorites, etc.

Subscription to the News Services (for each study the librarians create a weekly newsletter with a news selection e-mail)

Subscription to paper journal TOC

High

High

Medium

Look into exercises of other semesters 
There are more possible actions that are currently being performed in the context of the digital library scenario of use but that are not integrated yet. For instance, teachers usually recommend a basic bibliography for any subject, which is supposed to be used by students for solving the course exercises. The use of an ontology could be also interesting to incorporate such new functionalities into the existing digital library, by describing the relationships between elements. In plain words: if a teacher defines one or more books as recommended bibliography for a given subject, students enrolled in such subject should be aware of those books when performing searches related to the subject. Discovering these new functionalities requires from the study of the current user behavior (taking into account the different user profiles) in the virtual e-learning environment, by means of classical HCI methodologies (Dix et al., 1998), involving users in the design of the new digital library. Then, these functionalities and requirements can be described as rules ranging from simple to complex statements within the ontology framework.

For library managers, the creation of an ontology will help them to construct tailored libraries for each subject. Every library is built on the explicit recommendations from a teacher, but in an unstructured manner. With an ontology, these specialized libraries could be built from the use that previous students gave to that resources and new information could be added from the use of the library by experts. The ontology itself is composed of sub-ontologies. Thus, the digital resources, which are catalogued using the Dublin Core metadata or MARC format among other standards can be extended by means of an ontology to include additional information, automatically or in explicit way, such as their usage, user ratings and any other useful information that users might consider, such as summaries, enhanced taxonomies and keywords, for example.

\section{Privacy issues}

A very important aspect that cannot be ignored is the fact that users are always under control, in the sense that all taken actions are monitored and registered. This might seem a very invasive setup which harms user privacy and, therefore, undesirable. Nevertheless, there are several remarkable facts that need to be clarified:

- users know in advance that, in a virtual e-learning environment (or any other web based environment), all actions are logged;

- the recommendation system must be designed in a non-intrusive manner and be user-friendly, including the possibility of disconnecting it or minimizing its participation in the browsing or searching activities; and

- the participation of each individual user in the final recommendation system is completely anonymous.

Finally, it is also important to remark that the collected information is only used with personalization purposes, and it is not meant for commercial reasons, and that the library (a non-profit organization) will use the data rationally and in a transparent way. As usual, a tradeoff between personalization and privacy must be established. The more information the user reveals, the more personalized services he or she obtains.

On the other hand, the browsing and searching history of each user is part of his or her private profile, and only the user can modify (delete) his or her history records in order to update his or her preferences and navigational profile, according to the directions given by the personalization system. Some parts of this profile can be made 
LM

$26,4 / 5$ publicly available in order to create strong links with other users sharing the same navigational interests. There is evidence that people are sometimes eager to be identified and become part of a community (Lynch, 2003), and the use of ontologies could be one of the strengths of digital libraries for pursuing such purpose.

\section{4}

\section{Elements of a digital library ontology}

First, it is important to clarify that we are not building an ontology for describing the contents of a digital library, but an ontology for describing the way users browse and search such contents, with the aim of building a personalization system based on accurate recommendations. Therefore, more than building a low-level ontology for describing a particular concept, we are trying to describe a complex scenario of use. Table III describes the basic steps (Denny, 2002) that should be followed for describing the richness of such complex scenario. As usual, these steps overlap and must be taken in a recursive way: depending on the results of the evaluation undertaken in the fifth step, several definitions in the second and next steps might be modified. In fact, the ontology in itself will evolve with the new apparition of desired functionalities and requirements.

Acquiring domain knowledge and organizing the ontology, are where more efforts must be made to ensure a complete representation of the digital library scenario of use. As described above, Tables I and II outline the basic attributes and actions in the

\begin{tabular}{|c|c|c|}
\hline Step & Description & Resources/participants \\
\hline $\begin{array}{l}\text { Acquire domain } \\
\text { knowledge }\end{array}$ & $\begin{array}{l}\text { Assemble appropriate information } \\
\text { Define all terms used in the domain to describe } \\
\text { elements } \\
\text { Check formality and consistency }\end{array}$ & $\begin{array}{l}\text { Expertise from library } \\
\text { managers, computer scientists, } \\
\text { usability experts }\end{array}$ \\
\hline $\begin{array}{l}\text { Organize the } \\
\text { ontology }\end{array}$ & $\begin{array}{l}\text { Design overall conceptual structure } \\
\text { Identify domain's principal concrete concepts } \\
\text { and properties } \\
\text { Identify relationships among such concepts } \\
\text { Minimize possible overlaps and inconsistencies } \\
\text { Define actions performed between concepts }\end{array}$ & $\begin{array}{l}\text { Definitions: } \\
\text { user profiles } \\
\text { digital resources } \\
\text { navigational profiles } \\
\text { learning activities } \\
\text { Personalization system } \\
\text { functionalities }\end{array}$ \\
\hline $\begin{array}{l}\text { Elaborate the } \\
\text { ontology }\end{array}$ & $\begin{array}{l}\text { Add all the concepts, relations and individuals to } \\
\text { achieve a necessary level of detail } \\
\text { Reuse information from the current environment } \\
\text { as much as possible }\end{array}$ & $\begin{array}{l}\text { Instances of previously defined } \\
\text { concepts }\end{array}$ \\
\hline $\begin{array}{l}\text { Consistency } \\
\text { checking }\end{array}$ & $\begin{array}{l}\text { Reconcile syntactic, logical and semantic } \\
\text { inconsistencies } \\
\text { Involve automatic classification for defining new } \\
\text { concepts and class relationships }\end{array}$ & Ontology logging \\
\hline $\begin{array}{l}\text { Validate the } \\
\text { ontology }\end{array}$ & $\begin{array}{l}\text { Perform a final verification } \\
\text { Commit the ontology in a real operative scenario } \\
\text { of use } \\
\text { Compare the obtained results with the desired } \\
\text { functionalities } \\
\text { Detect all the possible mistakes or misuses of the } \\
\text { real scenario }\end{array}$ & $\begin{array}{l}\text { User tests } \\
\text { Data analysis } \\
\text { Ontology mining }\end{array}$ \\
\hline
\end{tabular}

Table III.

Basic steps for designing ontology 
current digital library and e-learning environments, which should be used as basic pieces for building the ontology.

Towards

personalization

\section{Organization of the digital library ontology}

Therefore, the ontology is built from the intersection of the elements in Tables I and II, by describing all the interesting relationships for recommendation purposes. This is carried out by defining small micro-scenarios which reveal typical uses of the digital library and their impact on user profile and navigational history. For instance, suppose the following micro-scenario: John is a researcher working in his $\mathrm{PhD}$ in machine learning, as his profile says. He is working with Mary, his advisor, who got her $\mathrm{PhD}$ in the same field. Mary is also the advisor of other two students, Peter and Ann, which are also working in machine learning related subjects. When students search for scientific papers, the results are sorted depending on papers' relative importance, according to the number of times each paper has been downloaded by other researchers (giving more importance to the other students which work with Ann) but also on whether such paper has been reviewed or not by any senior researcher (giving more importance to Ann's opinion).With the appropriate tools and ontology definition languages, such statement can be translated into one or more rules that feed the recommendation system with the actions taken by all the users in the digital library.

It is remarkable that the use of ontologies can be also extended to implement and transfer the concept of user profile and user navigational behavior to other digital libraries and databases, so when a digital library user leaves one service to connect into another one, the user profile (including preferences and navigational behavior) can be transferred from one database to another through the appropriate semantic web services, because all databases share a common domain of discourse that can be interpreted further by rules of inference and application logic.

Regarding implementation issues, ontologies are usually described by means of one or more descriptive languages based on XML (W3C, 2004a). Basically, RDF (W3C, 2004b) is used for describing resources, while DAML + OIL (W3C, 2004b) which is currently being evolved into the Web Ontology Language (OWL) standard (W3C, 2004c), is becoming the standard for describing ontologies and accessing resources through the web. The use of XML and description language standards ensures the interconnectivity with other existing ontologies and the possibility of upgrading the ontology for new requirements and functionalities. The widespread of XML for describing data (but also information and knowledge, with the help of ontologies) has made possible the apparition of new applications into classical areas of knowledge.

\section{Conclusions}

In this paper we have described the requisites of a personalization system which uses all information relevant to the process of searching and browsing a digital library to build a complete navigational profile for each user and its semantic description by means of an ontology. Then, all these profiles are then combined with the help of an ontology that establishes the possible relationships between all the elements present in a typical scenario of use in a digital library integrated in an e-learning environment. We have described the basic functionalities of the personalization system by means of use cases, and a methodology for building and ontology which describes the complete scenario of use. We have also identified the basic elements which are used to build such 
LM

$26,4 / 5$

216 ontology: user profiles, navigational profiles, user actions and the relationships established between these elements which are used by the personalization system.

Ontologies are a powerful tool for describing complex scenarios of use such as a digital library, where several concepts and relationships between these concepts can be identified and formally represented. The use of ontologies promotes the integration of new services into existing ones, and the interoperability with other systems through the appropriate semantic web services. New system functionalities and requirements can be added by including the appropriate description into the ontology framework that defines the digital library scenario of use.

Current and further research in this subject include the integration of the digital library personalization services with other personalization mechanisms provided by the virtual campus, towards a unique and complete user model. The digital library should become, therefore, another piece of the e-learning environment fully integrated into the learning process. The inclusion of new concepts related to the temporal validity of the ontology instances (resources, users and so) and their relationships should be also addressed. Finally, the definition of a validation rating algorithm combining both automatic but also user explicit rating systems is also under consideration.

\section{References}

Adams, K. (2002), "The Semantic Web: differentiating between taxonomies and ontologies", Online, Vol. 26 No. 4, pp. 20-3.

Adomavicius, G. and Tuzhilin, A. (1999), "Integrating user behavior and collaborative methods in recommender systems", Proceedings of the CHI'99 Workshop "Interacting with recommender systems", Pittsburgh, PA.

Arms, W.Y. (2002), Digital Libraries, The MIT Press, Cambridge, MA.

Brophy, P. (2001), The Library in the Twenty-First Century, Library Association Publishing, London.

Brophy, P. (2004), "Networked learning and networked information: towards a theoretical basis for the development of integrated information environment", Proceedings of the 4th Networked Learning Conference, Lancaster, UK, available at: www.shef.ac.uk/nlc2004/ Proceedings/Symposia/Symposium9/Brophy.htm

Callan, J. and Smeaton, A. (2003), "Personalization and recommender systems in digital libraries", Joint NSF-EU DELOS Working Group Report, available at: www.dli2.nsf.gov/ internationalprojects/working_group_reports/personalisation.html

Chen, H. (2003), "Towards building digital library as an institution of knowledge", NSF Post Digital Library Futures Workshop, Chatham, MA, available at: www.sis.pitt.edu/ $\%$ 7Edlwkshop/paper_chen.html

Colkin, E. (2001), "Personalization tools dig deeper”, Information Week, No. 852, pp. 49-51.

Denny, M. (2002), “Ontology building: a survey of editing tools”, xml.com, available at: www. xml.com/pub/a/2002/11/06/ontologies.html

Dix, A., Finlay, J., Abowd, G. and Beale, R. (1998), Human-Computer Interaction, 2nd ed., Prentice Hall, Englewood Cliffs, NJ.

Ford, N. and Ford, R. (1993), "Towards a cognitive theory of information accessing: an empirical study”, Information Processing \& Management, Vol. 29 No. 5, pp. 569-85. 
Gapen, D.K. (1993), "The virtual library: knowledge, society and the librarian", in Saunders, L. (Ed.), The Virtual Library Visions and Realities, Meckler Corporation, Westport, CT, pp. 1-14.

Gruber, T.R. (1995), "Toward principles for the design of ontologies used for knowledge sharing", International Journal of Human-Computer Studies, Vol. 43, pp. 907-28.

Herlocker, J.L., Konstan, J.A., Terveen, L.G. and Riedl, J.T. (2004), "Evaluating collaborative filtering recommender systems", ACM Transactions on Information Systems, Vol. 22 No. 1, pp. 5-53.

Jonassen, D., Davidson, M., Collins, M., Campbell, J. and Haag, B. (1995), “Constructivism and computer-mediated communication in distance education", The American Journal of Distance Education, Vol. 9 No. 2, pp. 17-23.

Kasanoff, B. (2001), Making it Personal, Perseus Publishing, Cambridge, MA.

Lynch, C. (2003), "Digital library opportunities”, Journal of Academic Librarianship, Vol. 29 No. 5, pp. 286-9.

Mor, E. and Minguillón, J. (2004), "E-learning Personalization based on Itineraries and long-term navigational behavior", Proceedings of the 13th International World Wide Web Conference, Alternate Track Papers and Posters, pp. 264-265.

Nielsen, J. (1998), "Personalization is over-rated", Alertbox. available at: www.useit.com/ alertbox/981004.html

Riecken, D. (2000), "Personalized views of personalization", Communications of the ACM, Vol. 43 No. 8, pp. 27-8.

Rosenberg, M.J.E-L.e. (2001), E-learning: Strategies for Delivering Knowledge in the Digital Age, McGraw-Hill, New York, NY.

Sadeh, T. and Walker, J. (2003), "Library portals: toward the Semantic Web", New Library World, Vol. 104 Nos. 1184/1185, pp. 11-19.

Schonberg, E., Cofino, T., Hoch, R., Podlaseck, M. and Spraragen, S.L. (2000), "Measuring success", Communications of the ACM, Vol. 43 No. 8, pp. 53-7.

Sheth, A., Ramakrishnan, C. and Thomas, C. (2005), "Semantics for the Semantic Web: the implicit, the formal and the powerful", International Journal on Semantic Web \& Information Systems, Vol. 1 No. 1, pp. 1-18.

Sicilia, M.A. and García, E. (2005), "On the convergence of formal ontologies and standardized e-learning”, Journal of Distance Education Technologies, Vol. 3 No. 12, pp. 12-28.

Sinha, R. and Swearingen, K. (2001), "Comparing recommendations made by online systems and friends", Proceedings of the Second DELOS Network of Excellence Workshop on Personalization and Recommender Systems in Digital Libraries, available at: www.ercim. org/publication/ws-proceedings/DelNoe02/RashmiSinha.pdf

W3C (2004a), eXtensible Markup Language (XML) 1.0 (Third Edition), available at: http://www. w3.org/TR/REC-xml/

W3C (2004b), Description Framework (RDF) Primer, available at: www.w3.org/TR/rdf-primer/

W3C (2004c), Ontology Language (OWL) Guide, available at: http://www.w3.org/TR/owl-guide/ 